\title{
ALGUNOS ASPECTOS DE LA EQUIVALENCIA IDIOMÁTICA EN FRANCÉS Y EN CASTELLANO
}

M." Rosario Ozaeta Gálvez

UNED

INTRODUCCIÓN

El presente artículo constituye la segunda parte de un trabajo anteriormente realizado en torno a la problemática de la traducción de las expresiones idiomáticas del francés al castellano, al que pretende completar, abordando distintos aspectos del mismo tema.

El referido estudio' perseguía una doble finalidad: por una parte, investigar en la terminología existente, con el fin de esclarecer sus límites, y por otra, tratar de demostrar la tesis de la traducibilidad de las expresiones idiomáticas. El primer objetivo se vio cumplido con muchas reservas; es destacable la obscuridad conceptual en este terreno; los límites entre unas y otras denominaciones son imprecisos. No obstante, tras un intento de franquear dichos límites en francés y en castellano mediante una revisión terminológica, efectuamos una opción; la denominación: locuciones y expresiones idiomátiças comprende, según nuestro criterio, todo el vasto abanico de este campo, cuya traducción a otra

1 "La traduction des locutions et des expressions idiomatiques», Cuadernos de Filologia Francesa, 5. Universidad de Extremadura. Cáceres, 1991, pp. 199-218. 
lengua juzgamos posible —cubriendo así el segundo objetivo-, por la vía de la equivalencia.

Nuestra propuesta de sistematización de los procedimientos de traducción parte, pues, del convencimiento de la viabilidad de la correspondencia en las mencionadas expresiones, dentro de diversos niveles de equivalencia, que hemos dividido en tres grupos: transferencia de la misma imagen; recreación de una imagen equivalente; pérdida de la imagen y, por consiguiente, de la idiomaticidad.

Deseamos en esta ocasión profundizar en algunos aspectos de la parcela relativamente inexplorada, aunque absolutamente apasionante, de la equivalencia idiomática, en la cual se pone en juego un buen número de factores que rebasan lo lingüístico, afectando en gran medida a la relación entre las culturas y las civilizaciones.

\section{CRITERIOS DE CLASIFICACIÓN}

Los intentos de clasificación de las expresiones idiomáticas han sido múltiples, lo que obedece a la dificultad de definición a la que ya hemos hecho referencia. Juzgamos de interés detenernos a presentar algunas de las clasificaciones que a nuestro juicio han constituido una aportación positiva en el terreno de la Fraseología.

Florica Dimitrescu ${ }^{2}$ distingue tres tipos de locuciones segün su grado de fusión, que relaciona directamente con su antigüedad en la lengua. Por su parte, A. V. Isačenko ${ }^{3}$ se basa en la distinción realizada por Vinogradov entre: groupes phraséologiques soudés, indivisibles e imposibles de descomponer; unités phraséologiques, o grupos de palabras fijas empleados en sentido figurado que el autor identifica a veces con los clichés-, y assemblages phraséologiques o clichés-phrases. Dicha clasificación se basa en el criterio de la fijación.

J. P. Vinay y J. Darbelnet, en su obra: Sylistique comparée du français et de l'anglais ${ }^{4}$, proponen una clasificación que tiene igualmente su origen en el

${ }^{2}$ En su estudio: «Le concept de locution», Mélanges linguistiques, VIIIle Congrès International des linguistes (Oslo, 5-9 de agosto de 1957). Éd. de l'Académie de la République populaire roumaine. Bucarest, 1957, pp. 269-289.

${ }^{3}$ En «Morphologie, syntaxe et phraséologie», Cahiers Ferdinand de Saussure. 7/1948. Genève, Droz, 1967, pp. 17-32.

4 Paris, Didier, 1977, p. 39 y ss. 
grado de cohesión de los elementos, entre groupes unifiés y groupements par affinité, y que encontramos poco precisa.

La contribución de E. Coseriu —sin ánimo de establecer juicios de valor, en este campo, poco fructíferos-, nos parece bastante más completa, clara, y por consiguiente, más enriquecedora. Nos referimos a su conocida distinción entre «técnica del discurso» y «discurso repetido». Según el autor:

La «técnica del discurso» abarca las unidades léxicas y gramaticales (lexemas, categoremas, morfemas) y las reglas para su modificación y combinación en la oración, es decir, las «palabras» y los instrumentos y procedimientos léxicos y gramaticales. El «discurso repetido» abarca todo lo que tradicionalmente está fijado como «expresión», «giro», «modismo», "frase» 0 «locución» y cuyos elementos constitutivos no son reemplazables o re-combinables según las reglas actuales de la lengua ${ }^{5}$.

Coseriu sintetiza en estas definiciones algunos trabajos anteriores que recogen una clasificación semejante ${ }^{6}$. Se trata, en suma, de la distinción entre las unidades complejas «à éléments variables et à éléments constants» ${ }^{7}, 0$, en otras palabras, entre la combinación libre y el grupo fraseológico, clasificación que ha gozado de una larga tradición en la lingüística europea.

El autor diferencia tres clases de unidades, según su grado de combinabilidad estructural y según los niveles en los que éstas son conmutables, y las denomina «locuciones», «sintagmas estereotipados» y «perífrasis léxicas». Aunque no acepta el análisis de la estructura interna de dichas expresiones - Coseriu afirma su significado «en bloque»-, se vislumbra un nuevo criterio, ya no formal, sino semántico, que se basa en la idiomaticidad.

Una distinción que nos parece particularmente coherente, es la que establece Alberto Zuloaga en su obra: Introducción al estudio de las expresiones fijas $^{8}$, al considerar dos criterios que juzgamos han de ser tenidos en cuenta

5 Coseriu, E. Principios de Semántica Estructural. Versión española de Marcos Martínez. Madrid, Gredos, 1986 (1977), Biblioteca Románica Hispánica. II. Estudios y Ensayos, 259, p. 113.

- Lo que pone de manifiesto A. Zuloaga en su obra: Introducción al estudio de las expresiones fijas. Frankfurt. Peter D. Lang, 1980, Studia Romanica et Linguistica, 10, p. 77 y ss. Se cita como ejemplo a H. Paul, Ch. Bally, C. de Boer, y otros.

7 Según la terminología de J. Dubois, en «Les notions d'unité sémantique complexe et de neutralisation dans le lexique», Cahiers de lexicologie, Vol. 2, 1960; Paris, Didier, pp. 62-66.

8 Op. cit., p. 95 y ss. 
necesariamente en la identificación y análisis de las unidades idiomáticas, con vistas a su adecuada traducción posterior. Se trata de la fijación y de la idiomaticidad. El autor lleva a cabo una doble clasificación de las expresiones fijas; la primera de ellas incluye las expresiones según los rasgos de su estructura interna - siguiendo los mencionados criterios de fijación e idiomaticidad-. La segunda clasificación recoge el valor funcional de las unidades.

Presentamos seguidamente, por considerarlos de interés, los esquemas correspondientes a dichas clasificaciones, ligeramente simplificados en pro de una mayor claridad, e ilustrados con algunos ejemplos en francés.

Clasificación según la estructura interna

Rasgos fundamentales: fijación-idiomaticidad

Tipos de expresiones:

1. Combinaciones libres

2. Combinaciones fijas (sentido puramente literal): Aussitôt dit, aussitôt fait

3. Combinaciones semiidiomáticas (motivación parcial): C'est bien fait

4. Combinaciones idiomáticas (carencia de autonomía semántica): À tort et à travers Se payer la tête de quelqu'un

- Mixtas (una parte fija y otra idiomática): Vivre comme un coq en pâte Ne pas avoir froid aux yeux

- Idiomáticas con elementos únicos: Voilà le hic À la queue leu leu

- Idiomáticas con anomalías estructurales: Sans coup férir À son corps défendant 
- Idiomáticas con elementos metalinguííticos o autodesignativos: Un tiens vaut mieux que deux tu l'auras

- Idiomáticas susceptibles de presentar en el discurso una expresión homófona en sentido literal: Rompre (briser) la glace

Clasificación según el valor funcional

LOCUCIONES: Funcionalmente inferiores a la oración Requieren un contexto verbal inmediato

1. Equivalentes a unidades gramaticales:

- Prepositivas À la merci de, faute de

- Conjuntivas Malgré que, de peur que

- Elativas Comme quatre, comme un fou

2. Equivalentes a unidades léxicas, a sintagmas:

- Nominales Bouc émissaire, mauvais coucheur, gros bonnet

- Adnominales (adjetivas) Sain et sauf, malheureux comme une pierre

- Adverbiales De plus belle, la corde au cou, en cachette

- Verbales - Tirer la couverture à soi, prendre la poudre d'escampette 
ENUNCIADOS: Equivalentes o superiores a la frase

No requieren un contexto verbal inmediato para constituir una expresión de sentido completo en el habla.

\author{
1. Frases: \\ Clichés \\ Fórmulas \\ Dichos, frases hechas
}

2. Textos (refranes)

\title{
2. CARACTERES IDIOMÁTICOS
}

Debido a la naturaleza y objetivos de este estudio, se hace necesaria la exposición de algunas características básicas de las expresiones idiomáticas. No nos vamos a detener en los rasgos, de sobra conocidos, de unidad formal y de sentido, o de transgresión de las normas gramaticales o léxicas. Dejando a un lado estos caracteres digamos primarios, es digno de destacar el alto grado de expresividad, así como el aspecto comunicativo de las locuciones. La espontaneidad es otro factor fundamental, sobre todo con respecto al lenguaje familiar, terreno muy prolífico en expresiones de este género.

En cuanto al valor metafórico, éste constituye un rasgo esencial, aunque no se trata de un criterio definitorio: la mayoría de locuciones son metafóricas, pero no todas ellas lo son; no obstante, se puede afirmar que la metáfora es uncomponente básico de las expresiones idiomáticas, lo que justifica su gran riqueza. A. Rey afirma en el Prólogo de su Dictionnaire des Expressions et Locutions:

L'interêt de la métaphore en contraste avec les sens figés et séparés de leur origine... est qu'elle met en rapport deux réalités liées par une relation analogique sans abandonner la première. À côté de sa nouvelle valeur, qui correspond à des désignations stables..., le mot métaphorisé évoque, suggère, si peu que ce soit, si bizarrement que ce soit, sa première fonction?.

9 Paris, Les usuels du Robert, 1986, p. X. 
P. Guiraud reconoce igualmente la tendencia de las locuciones a conservar su motivación inicial, no sin apuntar la salvedad de que, en la mayoría de los casos, la imagen liberada por la locución se actualiza sin revelar el vínculo semántico que motivó la expresión:

...car les mots qui la composent, bien que formant une unité, gardent une certaine autonomie, et continuent à évoquer des images qui leur sont propres $^{10}$.

J. Casares considera una condición indispensable para la identificación de las expresiones el hecho de que el contenido semántico mantenga una determinada virtualidad, subsistiendo así en la conciencia de la comunidad lingüística" ${ }^{11}$.

La motivación de las expresiones idiomáticas ha sido siempre un terreno muy espinoso, y por ello, objeto de gran polémica. Algunas expresiones son menos motivadas que otras, en cuyo caso, la etimología popular desempeña un papel básico en cuanto a su remotivación. Por otra parte, como señala A. Rey:

Les lois générales de la «démotivation», de l'oubli des origines... sont combattues, en matière de phraséologie, par des effets de sens proliférants, nés du contact entre la connaissance des mots et l'imaginaire ${ }^{12}$.

La delimitación del grado de motivación resulta bastante ardua. A. Zuloaga, que llega a defender una doble arbitrariedad - natural y relativa-, basándose en la arbitrariedad del signo, sólo admite la existencia de cierto grado de motivación cuando la relación entre ambos significados —el literal y el idiomá-

10 Guiraud. P. Les locutions françaises. Paris, PUF, 1962, pp. 7-8.

11 El autor realiza un estudio exhaustivo de las locuciones y de los modismos delimitando y caracterizando con gran claridad ambos conceptos, en su obra: Introducción a la lexicografía moderna. Revista de Filología Española. Anejo LII. Madrid, Consejo Sup. de Investigaciones Científicas, Patronato «Menéndez y Pelayo», Instituto «Miguel de Cervantes», 1969 (3.‘ ed. 1992), páginas 205-242.

12 Op. cit., p. XI. 
tico- es evidente desde el punto de vista del funcionamiento real de la expresión, y aun entonces, el autor afirma el claro predominio de la arbitrariedad de la relación entre los dos sentidos ${ }^{13}$.

En dichos casos, conviene establecer una clara distinción entre las expresiones idiomáticas no motivables y las que sí lo son, dependiendo del contexto el funcionamiento literal de estas últimas. Según C. Kerbrat-Orecchioni, uno de los procedimientos más productivos para formar un juego de palabras es el de explotar un hecho de lexicalización consistente en mantener a la vez el valor propio y figurado de una expresión fija. La autora da como ejemplo algunos eslóganes en los que se produce frecuentemente este fenómeno:

«Montrez les dents: Ultrabrite»

«Mettez-leur l'eau à la bouche: Perrier» ${ }^{14}$

Por nuestra parte, reconocemos un aspecto variable en las expresiones, cuyos valores connotativos $-\mathrm{y}$ en consecuencia, las posibles motivaciones y remotivaciones-, dependen de determinados factores que J. Casares ha especificado con gran precisión al abordar las eventuales influencias en el uso del modismo ${ }^{15}$. Dichos factores pueden ser o bien externos, relacionados con la idiosincrasia del hablante - se trata de coordenadas sociolingüísticas como el nivel cultural, la edad, la intención o la situación-, o bien intrínsecos, como es la virtualidad para producir determinados efectos estilísticos. El conocimiento y uso de las locuciones es muchas veces producto del azar y depende en gran medida del hablante.

Esta concepción nos remite necesariamente al carácter de inalterabilidad de las expresiones, criterio que no es en absoluto definitivo, si se tiene en cuenta la existencia real de variantes. A. Zuloaga no admite ni las variantes diatópicas, ni las diastráticas o diafásicas. Según su teoría, las verdaderas variantes, independientes del contexto, han de ser parcialmente idénticas en cuanto a su estructura y componentes, dentro de la misma lengua funcional. Nuestro criterio difiere en este punto. Afirmamos la existencia de variantes; en primer lugar, variantes estilísticas. B. Lafleur caracteriza así a las locuciones en su Dictionnaire des locutions idiomatiques franf̧aises:

13 Op. cit., p. 126 y ss.

14 Kerbrat-Orecchion, C. La connotation. Presses Universitaires de Lyon, 1977, p. 144.

15 En el estudio anteriormente mencionado, que se incluye en op. cit. 
Elles sont assises entre deux chaises: celle de la langue et celle du style ${ }^{16}$.

Existen además otras variantes según los distintos estratos socio-culturales, que pueden variar a su vez según las épocas. Finalmente, se pueden detectar variantes según la vitalidad de las expresiones a través del tiempo, lo que está en el origen de la habitual distinción entre locuciones en desuso, o de uso corriente.

Dejamos en este punto la consideración de otros caracteres, para tratar brevemente de un fenómeno que aparece en múltiples ocasiones al abordar el estudio de las expresiones idiomáticas.

\section{El ASPECTO FóNICO}

Es nuestro propósito poner de relieve el papel desempeñado por algunos aspectos de la forma fónica de las expresiones. H. Chuquet y $\mathbf{M}$. Paillard afirman en su obra Approche linguistique des problèmes de traduction:

...Le parallélisme prosodique joue souvent un rôle de renforcement ou de substitut de la métaphore dans la traduction et... ils fonctionnent l'un et l'autre, sur deux plans différents, selon le principe d'évocation par similarité. La correspondance est particulièrement manifeste dans la traduction des proverbes et expressions... ${ }^{17}$.

La eficacia de los recursos fónicos es particularmente evidente en numerosas expresiones. Vamos a exponer algunas de ellas, junto a su traducción, así como los procedimientos empleados.

Los ejemplos de aliteraciones son numerosos. La locución adjetiva: Entre le zist et le zest encuentra una fiel correspondencia en su equivalente: «ni fu ni fa».

16 Éd. du Renouveau pédagogique, Ottawa, Canada, 1991 (1979), p. XVI.

17 Paris, Ophrys, 1989 (1987), p. 29. 
Sin embargo, en la expresión: Ménager la chèvre et le chou, el efecto de aliteración se pierde en la traducción: «saber nadar y guardar la ropa». Dicha expresión ha dado origen a otra más actual, empleada en el léxico de la política: une motion chèvre-chou, para calificar a una moción que trata de contentar a todos.

La aliteración, esta vez mantenida en castellano: Boucler la boucle, «rizar el rizo», se da igualmente en inglés: To loop the loop.

Existen otros procedimientos, como es el caso de algunas expresiones cuyos elementos están rimados, por ejemplo: Des espèces sonnantes et trébuz chantes, y su traducción "paralela»: «dinero contante y sonante». A veces, la rima es asonante, como en la locución: Par monts et par vaux; en esta ocasión, el castellano expresa la misma noción de movimiento por medio de la rima consonante: «de la Ceca a la Meca».

El efecto fónico puede consistir en una gradación silábica: À bâtons rompus (a-a-õ-o--y), expresión que se traduce recurriendo a la aliteración: «sin ton ni son», y también manteniendo la gradación de sílabas: «sin orden ni concierto» (i-o-e-i-o-i-e-o).

En ocasiones, los componentes se repiten, como por ejemplo, en la locución, muy frecuente en francés: Donnant donnant, que se traduce por: «toma y daca», expresión de estructura claramente rítmica.

Citaremos por último la yuxtaposición estilística, recurso muy utilizado, que consiste en un emparejamiento de sustantivos a menudo sinónimos. Este procedimiento fue empleado habitualmente en la Retórica tradicional, sobre todo durante la Edad Media. Los ejemplos son numerosos:

Les us et coutumes: «los usos y costumbres»

Con frecuencia se puede apreciar un efecto de aliteración añadido en estas parejas de términos, que a veces es paralelo en la traducción castellana: Sans rime ni raison, sain et sauf, bel et bien, peu ou prou...

A. Zuloaga, en su excurso dedicado a las «figuras fónicas en unidades fraseologicas» ${ }^{18}$, subraya la importancia que encierran dichos efectos fónicos; según el autor, la rima, las aliteraciones, las estructuras rítmicas... no se emplean en las expresiones de un modo fortuito. Por el contrario, los procedimientos

18 En op. cit., p. 115 y ss. 
mencionados actúan de refuerzo en la cohesión de las unidades, acentuando la fijación del texto, y contribuyen a generalizar en la norma linguiística social estas combinaciones idiomáticas, cuya función lúdica no es tampoco desdeñable. P. Guiraud señala al respecto:

...les locutions qui forment la partie la plus singulière d'une langue... ne sont le plus souvent que le résultat d'accidents formels qu'exploitent la naìveté ou la malice du langage ${ }^{19}$.

\section{ALGUNOS TIPOS ESPECÍFICOS DE EQUIVALENCIA IDIOMÁTICA}

Ya se ha mencionado al inicio del presente artículo la existencia de tres niveles de equivalencia en el terreno de las expresiones idiomáticas, tomando como puntos de referencia al francés y al castellano. Pues bien, dentro del segundo grupo, al que denominamos «de equivalencia idiomática», pretendemos destacar algunos campos específicos que entrañan un especial interés. En este nivel de equivalencia tiene lugar una recreación de la imagen contenida en la expresión, lo que a su vez se lleva a efecto según diversos grados, unos más próximos, otros más alejados, con respecto a las dos lenguas de referencia.

\subsection{Expresiones de contenido pragmático y cultural}

El conocimiento de algunas expresiones que encierran un valor pragmático, reflejando una situación de la realidad social, reviste una gran importancia con vistas a la reproducción de la equivalencia exacta, muchas veces muy distante en cada civilización. Así, Coiffer sainte Catherine se traduce por: «quedarse para vestir santos». L'été de la Saint-Martin (en Canadá: l'été indien), época de los buenos días soleados ya entrado el otoño, se traduce frecuentemente por: «el veranillo de san Miguel», llevando a cabo un desplazamiento

19 Guiraud, P. Op. cit., p. 106. 
temporal exigido por el santoral correspondiente, que a su vez responde a la diversidad climatológica.

Poisson d'avril equivale en castellano a una «inocentada», que se practica en el mes de diciembre.

Existen muchas otras fórmulas de la vida cotidiana, juegos, lenguaje infantil... de las que damos algunos ejemplos:

Brigadier, vous avez raison! - «Lo dijo Blas, punto redondo»

Escargot, montre-moi tes cornes! - «Caracol, saca los cuernos al sol»

La conocida fórmula de los prestidigitadores: Rien dans les mains, rien dans les poches, pasa a ser: «nada por aquí, nada por allá»; La poudre de perlimpinpin, «los polvos de la madre Celestina»...

Por otra parte, si consideramos su origen, muchas locuciones son alusiones textuales. P. Guiraud afirma que la lengua crea a veces personajes, hechos imaginarios, que los escritores desarrollan, actualizando las virtualidades de sus imágenes, latentes en las palabras. Dice así:

Au commencement était le Verbe; puis sont venus les poètes qui ont donné des choses aux noms; enfin sont venus les linguistes qui ont tout embrouille ${ }^{20}$.

De entre las numerosas referencias culturales, algunas pertenecen a la Antigüedad griega o latina; otras, a la Literatura, a la Biblia o a la Historia.

La expresión: Se croire sorti de la cuisse de Jupiter tiene una variante «histórica»: Être sorti de la côte de Charlemagne (existe otra variante, más antigua: Être de la côte de Saint Louis). En castellano, el carácter histórico se ha mantenido en la expresión: «creerse descendiente de la pata del Cid».

Sin embargo, la réplica: Qu'allait-il faire dans cette galère?, perteneciente a Les Fourberies de Scapin, pierde la imagen literaria en la traducción que, por otra parte, encierra una gran expresividad: «¿Quién le mandó meterse en ese berenjenal?»

La expresión siguiente acusa igualmente una pérdida de la imagen literaria en castellano: Revenir à ses moutons, frase de la famosa Farce de Maître Pathelin, que se traduce sencillamente por: «a lo que íbamos» (Revenons...)

20 Ibid., p. 104. 
Faire comme les moutons de Panurge es un caso distinto de equivalencia, ya que corresponde a una fórmula proverbial: «¿Dónde va Vicente...?

El carácter cultural, histórico o literario es, pues, mantenido en contadas ocasiones. No obstante, otra imagen surge, más o menos expresiva -es esta una valoración altamente subjetiva-, pero siempre de gran viveza.

\subsection{Expresiones de contenido simbólico}

Existen algunas expresiones que encierran un determinado simbolismo $y$ que constituyen un campo muy sugerente. Concretamente, nos estamos refiriendo al simbolismo numerológico. Son frecuentes los casos en los que el valor de las cifras entraña un determinado significado, que vamos a considerar desde la optica de la equivalencia.

El número quatre forma parte de numerosas expresiones, y designa tanto a una pequeña cantidad: Quatre pelés et un tondu, en castellano: «cuatro gatos»; À quatre pas: «a dos pasos»..., como a una pluralidad, llegando a desempeñar a menudo el papel de intensivo. Los siguientes ejemplos dan prueba de ello.

Avoir de l'esprit comme quatre, lo que viene a significar: «être très intelligent, fin et amusant», se puede traducir por la expresión: «tener la gracia por arrobas».

Habitualmente, el intensivo comme quatre, con verbos como manger, boire... significa «beaucoup». El intensivo equivalente en castellano es «siete» - Por ejemplo, en la frase: «comer más que siete»-.

En la locución: Se mettre en quatre, el valor de la cifra es igualmente intensivo, y se traduce por: «desvivirse por alguien».

Dire à quelqu'un ses quatre vérités: «decirle a uno cuatro verdades», «cantarle a uno las cuarenta».

En otras ocasiones se pueden apreciar connotaciones de agitación y de rapidez. Faire le diable à quatre, según el diccionario: «Faire beaucoup de bruit en s'agitant, s'agiter, se démener...», equivale a las expresiones castellanas: «armar la Marimorena, la de Dios es Cristo».

Ne pas y aller par quatre chemins se traduce por: «no andarse con rodeos, ir al grano».

A veces los empleos revisten un carácter más general: 
Tiré à quatre épingles: «ir de punta en blanco», «de tiros largos», es una expresión próxima a: Se mettre sur son trente-et-un, de origen obscuro, y también de valor intensivo.

La locución: Faire les quatre volontés de quelqu'un (ses quatre...), tiene una variante: Faire ses trente-six volontés..., en castellano, «hacer su santa voluntad». Aparece una nueva cifra, trente-six, tradicionalmente simbólica, y cuyo valor general es igualmente intensivo. Así, decimos:

Voir trente-six chandelles: «ver las estrellas»

Trente-six fois: $«$ cien, mil veces» 21

Otras cifras encierran a su vez un significado semejante. El número sept, cuyo valor intensivo en castellano ya ha sido señalado, posee un carácter marcadamente simbólico:

Tourner sept fois sa langue dans sa bouche se asemeja a: $Y$ regarder à deux fois, «pensárselo dos veces».

Répéter, recommencer... dix fois la même chose: «...cien, mil veces».

Des mille et des cents: «miles, millones».

A modo de resumen del presente apartado, podemos obtener dos claras conclusiones; por una parte, la competencia entre números «redondos» (cien, diez, mil) y cifras de valor simbólico, lo que, según el criterio de $\mathrm{A}$. Rey y de $\mathrm{S}$. Chantreau ${ }^{22}$ respondería a la substitución de los valores heredados por la economía aritmética. Por otra parte, el valor cuantitativo se ha revelado distinto, siendo por lo general más acusado en castellano que en francés.

5. UN EJERCICIO DIDÁCTICO: LOS «ESQUEMAS IDIOMÁTICOS»

Existen algunas estructuras semánticas, cuyos campos se encuentran claramente delimitados, que van a permitimos obtener un equivalente más próximo,

21 El número treinta y seis es uno de los frecuentes vestigios de nuestras lenguas, que atestiguan una antigua numeración por docenas -en este caso, tres veces doce-.

22 En su Dictionnaire des Expressions et Locutions. Paris, Le Robert, 1986, p. 332. Esta obra, citada anteriormente, nos ha servido de gran ayuda en la selección de las expresiones y de los rasgos descriptivos de éstas. 
siendo su tratamiento bastante más sencillo que el de otras combinaciones idiomáticas.

Nuestra propuesta consiste en la elaboración de esquemas muy simples, de modo que las áreas de los diferentes dominios considerados coincidan - al menos parcialmente-, y la traducción pueda realizarse siguiendo los rasgos señalados en cada semantismo concreto. Como señala A. J. Greimas:

...la comparaison ne doit pas être globale; elle doit s'établir à des niveaux $d$ 'analyse plus bas où elle peut trouver des éléments structurels directs et de ce fait comparables ${ }^{23}$.

Tras considerar distintos terrenos, nuestro análisis se ha centrado en el de la caracterización, tanto física como psicológica, hallando algunos contrastes sorprendentes, que creemos merece la pena mencionar.

Le lapin representa en francés al animal corredor por excelencia, mientras que en castellano es «el gamo», o bien «la liebre». El aburrimiento está simbolizado por un rat mort, une croûte de pain, une carpe - animal que representa igualmente al silencio-... en castellano, «la ostra» cumple idéntica función. En el ámbito francés, se toca un instrumento comme un dieu. En castellano, "como los (propios) ángeles». Los mismos ángeles simbolizan la paciencia en la lengua francesa; en castellano, es «el santo Job» quien se adorna con dicha virtud; por otra parte, también Job es símbolo de pobreza en francés...

La traducción de parler comme un moulin es «hablar por los codos», en tanto que el animal charlatán es «la cotorra» - une pie, en francés-. La falsedad está simbolizada por un jeton; en castellano, adoptando un rasgo de animación, es Judas su máximo representante. Laid comme les sept péchés capitaux se puede traducir, mediante un juego metonímico, por «más feo que pegar a un padre».

Los ejemplos son interminables. Se trata, en suma, de toda una caracterización psicológica, que multiplica las variantes de un semantismo común.

En lo que se refiere a su aspecto formal, los esquemas idiomáticos comprenden una serie de locuciones comparativas - verbales y adjetivas-, de acusada frecuencia de uso en las lenguas, y que en ocasiones vienen precedidas del

23 «Idiotismes, proverbes, dictons», en Cahiers de Lexicologie, Vol. 2, 1960, Paris, Didier, p. 46 . 
carácter global, a fin de lograr una descripción general previa que facilite y complete la comprensión y posterior equivalencia.

A. Zuloaga denomina elativas a estas locuciones, basándose en su efecto intensificador, y las describe en los siguientes términos:

La abrumadora mayoría de las locuciones elativas se presentan fijadas a un solo lexema, adjetivo o verbo, formando con él una unidad fraseológica del tipo que hemos llamado mixtas (con una parte idiomática o semiidiomática y otra meramente fija)... la parte idiomática o semiidiomática constituye el instrumento de elativización y la parte meramente fraseológica constituye el valor categorial y léxico de toda la unidad ${ }^{24}$.

Según nuestro criterio, no todas las locuciones comparativas presentan el mismo grado elativizador, siendo éste más neutro en algunas de ellas, que ostentan un carácter más marcadamente mimético e identificador. Por otra parte, destacamos la alta frecuencia del esquema: $x$ comme $y$ (adj. + comme $+\mathrm{SN}$ ) en francés, lengua que utiliza preferentemente el comparativo de igualdad, en contraste con el castellano, que ofrece una mayor frecuencia de la variante mayorativa: más $x$ que $y$.

Queda fuera de duda la posibilidad de ampliar el número de expresiones susceptibles de una esquematización que, por otra parte, no se limita tan sólo a las locuciones comparativas, pudiendo aplicarse igualmente a otros semantismos.

No se ha efectuado una selección previa de rasgos distintivos, lo que justifica su diversidad; cada esquema requiere unos semas específicos o genéricos, según el semantismo considerado. Somos conscientes de la dificultad que entraña cualquier intento de estructuración léxica, lo que se acentúa sensiblemente en el terreno fraseologico, a causa de la relatividad impuesta por la base extralingüística que subyace a dichos rasgos, los cuales, en este caso, deben considerarse como meros puntos de referencia orientados a servir de puente hacia una equivalencia más cercana.

Presentamos, por fin, algunos esquemas, cuya pretensión no va más allá de una eventual utilización didáctica, así como de una ilustración práctica del último apartado del presente artículo.

24 Zuloaga, A. Op. cit., p. 147. 


\section{APÉNDICE}

PAUVRETÉ Criterio: + o - metafórico. La traducción puede realizarse siguiendo este criterio y el contexto.

\begin{tabular}{|c|c|c|c|}
\hline $\begin{array}{c}\text { metáfora } \\
+ \\
\text { elaborada }\end{array}$ & $\begin{array}{l}\text { Tirer le diable par la queue } \\
\text { Traîner la savate } \\
\text { Être fauché comme les blés } \\
\text { Être dans la purée } \\
\text { Être à la côte } \\
\text { Etre sur le sable } \\
\text { Être à sec }\end{array}$ & Estar: & $\begin{array}{l}\text { a la cuarta pregunta } \\
\text { a dos velas } \\
\text { en las últimas } \\
\text { pelado } \\
\text { tronado }\end{array}$ \\
\hline
\end{tabular}

metáfora $\left\{\begin{array}{l}\text { Sans un radis } \\ \text { N'avoir pas un rond } \\ \text { N'avoir pas un rotin } \\ \text { Être sans le sou } \\ \text { Ne pas avoir un } \\ \text { sou vaillant }\end{array}\right.$

Sin una gorda, sin cinco, Sin un duro, céntimo, chavo, real, cuarto, lata, Sin blanca, sin cinco céntimos Sin una perra, peseta... monedas $\sin$ valor
Être dans le, en plein goudron dans la melasse dans la panade sur la paille
En la miseria

Estar con la lengua fuera

Pasar las de Caín
En la miseria

Tirer la langue

Manger de la vache enragée

\}

Être sans feu ni lieu sur le pavé

N'avoir pas une pierre où reposer sa tête
Estar en el arroyo

No tener donde caerse muerto 
PAUVRE comme...

Job

Être gueux comme un rat $\left.\begin{array}{c}\text { metáfora } \\ + \\ \text { elaborada }\end{array}\right\}$
Más pobre que...

Carracuca (=animado humano)

Las ratas (=animado no humano)

Raide comme un passe-lacet

Un maestro de escuela (+animado)

PARESSE Criterio: semantismo

Ne pas se faire des ampoules

Ne pas se fouler la rate

Ne pas en ficher un clou

\}

No herniarse

No pegar clavo

Avoir la cosse, tirer sa cosse

Avoir la rame

\}

)

S'endormir sur la manche

Se tourner les pouces (se les rouler)

Avoir les côtes en long

Tirer au flanc

Ne rien faire de ses dix doigts

Tirer sa flemme

Ne pas en ficher une rame, une ramée

Avoir un poil dans la main
Tener galbana

Estar mano sobre mano, de brazos cruzados

Tumbarse a la bartola

Escurrir el bulto

No dar golpe

Ser más vago que la chaqueta de un guardia, quinto 
Animales de escasa movilidad:

- Permanecen al sol $\quad\left\{\begin{array}{l}\text { une couleuvre } \\ \text { un lézard }\end{array}\right.$ - Se mueven lentamente $\left\{\begin{array}{ll}\text { une loche } \\ \text { une limace }\end{array} \quad\right.$ (- animado)

La chaqueta de un guardia, quinto

- Hibernan un loir

BÊTISE Criterio: semantismo

$\left.\begin{array}{ll}\text { Bête: à manger des chardons } & \text { à manger du foin }\end{array}\right\} \begin{aligned} & \text { tonto de capirote, de remate... } \\ & \text { (ver locuciones comparativas) }\end{aligned}$

En avoir, en tenir une couche $\left\{\begin{array}{l}\text { Ser un tontaina } \\ \text { Faltarle a uno un agua }\end{array}\right.$

Bouché à l'émeri......

Duro de mollera

Ne pas avoir inventé le fil à couper le beurre

Ne pas avoir inventé l'eau chaude (tiède, bouillie)

$\mathrm{Ne}$ pas avoir inventé la poudre

Enfoncer une porte ouverte

Faire le Jacques.

Gros Jean
No haber inventado la pólvora

Descubrir América

Hacer el tonto

Juan Lanas 


\section{BÊTE comme...}

Nombre de animal: un âne (bâté)..................... $\left\{\begin{array}{l}\text { Asno, borrico, rocín, animal, } \\ \text { burro, zopenco }\end{array}\right.$

une oie

Pavo, ganso, pavitonto, pavisoso

un cochon

Nombre de objeto:

une cruche

(alma) de cántaro

un panier

une valise, id. sans poignée

une malle

adoquín

1

Parte del cuerpo: ses pieds

Reino vegetal:

chou
un prunier (en desuso)

Cebollino, alcornoque calabaza, lila, melón

Loc. comparativa: más tonto que una mata de habas

Dominio abstracto: con comme la lune

Caer de un guindo

(tomber de la lune)

(一abstracto)

Loc. prov.: Il est fin comme Gribouille (qui se Más tonto que el que asó la jette dans l'eau par crainte de la pluie) manteca, que Abundio (=animado humano)

\section{DORMIR comme...}

DORMIR como...

Rasgos que caracterizan al sueño:

$\left.\begin{array}{ll}\text { - Apacible (animado): } & \begin{array}{l}\text { un ange } \\ \text { un bienheureux }\end{array}\end{array}\right\} \ldots . . . \quad$ un ángel (ito)

- Profundo (animales en hibernación):

un loir

un lirón

une marmote

una marmota 
- Pesado (objeto inanimado):

une bache, une souche

un plomb

(sommeil de plomb)

un pieu (¡verticalidad!) un leño, un tronco una piedra (sueño pesado)

trompo

un sabot

un sonneur (+animado)

Loc. adv.: À poings fermés

a pierna suelta

PENSAR como... (razonar)

RAISONNER comme...

- Animado humano: une femme soule

- Animado no humano: une bourrique un cheval de carrosse

un mosquito

- Inanimado:

une pantoufle

un coffre

un tambour (crevé)

DROIT, RAIDE comme...

Más derecho, recto, tieso que...

Concreto:

- Material, inanimado- un cierge

una vela

une canne.

un palo, una vara

un pieu
un piquet...
un I

un huso

- Mundo vegetal-

$\left.\begin{array}{l}\text { un jonc } \\ \text { un sapin }\end{array}\right\}$

un ajo

Abstracto:

la justice

la mort 
GRAS, GROS comme...

Más gordo que...

$\left.\begin{array}{ll}\text { - Animado humano: } & \text { un moine } \\ \text { un chanoine } \\ \text { un chantre }\end{array}\right\}$

- Animado no humano: une caille un cochon un porc

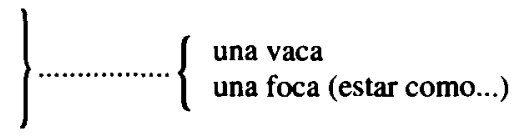

- Inanimado: $\quad-\quad\left\{\begin{array}{l}\text { un tonel (estar como...) } \\ \text { una mesa camilla (estar como...) }\end{array}\right.$

GAI comme...

- Animado humano: -

- Animado no humano: un pinson

- Inaminado:

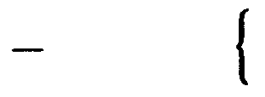

- Abstracto:

ROUGE comme...
Más contento que... (alegre)

un como chico con zapatos nuevos (estar)

unas castañuelas un cascabel (ser - lexicalizado-)

unas Pascuas

Valores expresados: vergüenza, pudor, emoción, cólera, causa física...

- Reino animal: Más rojo, colorado, que...

$\operatorname{coq}$

pavo

écrevisse homard cangrejo (ponerse como...)

- Reino vegetal:

coquelicot....................... amapola
pivoine
cerise
tomate ........................... tomate
carotte

Rougir comme... 
Rougir jusqu'au blanc des yeux

à la racine des cheveux

hasta las orejas

Piquer un fard, un soleil

subirse el pavo

BLANC, PÂle comme...

Más blanco que...

- Aspecto, color de la piel:

\begin{tabular}{|c|c|}
\hline $\begin{array}{l}\text { un cachet d' } \\
\text { un (pied de) }\end{array}$ & \}$_{\text {tbo }}^{\text {rine }}$ \\
\hline & \\
\hline
\end{tabular}

- Causa: enfermedad, miedo

\begin{tabular}{|c|c|}
\hline $\begin{array}{l}\text { un linge } \\
\text { un drap }\end{array}$ & \} \\
\hline
\end{tabular}

$\left.\begin{array}{l}\text { un cadavre } \\ \text { un, la mort }\end{array}\right\}$ 